\title{
Ribosomal-RNA patterns of Escherichia coli, Salmonella typhimurium and related Enterobacteriaceae
}

\author{
N. H. SMITH, PAMELA B. CRICHTON*, D. C. OLD* and C. F. HIGGINS
}

\section{Departments of Biochemistry and *Medical Microbiology, The University of Dundee, Dundee DD1 4HN}

\begin{abstract}
Summary. rRNA sequences are usually highly conserved among species. In Enterobacteriaceae we have shown that Salmonella typhimurium does not have an equivalent to the 23S rRNA of Escherichia coli but its 23S rRNA is cleaved in vivo into two smaller species. This cleavage appears to be a result of a difference between the $S$. typhimurium and $E$. coli rRNA sequences rather than to differences in ribonuclease activity. We have surveyed a wide range of Enterobacteriaceae for the presence or absence of 23S rRNA and found this rRNA species to be present in all strains of E. coli, Shigella and Citrobacter and all salmonellae examined except $S$. typhimurium. All $S$. typhimurium cultures, isolated at different times and from several different countries, lack an intact $23 \mathrm{~S}$ rRNA. Thus, the presence or absence of this rRNA species is an excellent diagnostic characteristic for $S$. typhimurium.
\end{abstract}

\section{Introduction}

Although different genera of the family Enterobacteriaceae often show only distant genetic and phenetic relatedness, several genes, such as those specifying the ribosomal RNAs, are highly conserved among species (Kohne, 1968; Brenner and Falkow, 1971). Thus, the isolated genes specifying rRNA show $\geqslant 90 \%$ relatedness by hybridisation analysis among species such as Escherichia coli and Proteus mirabilis (Kohne, 1968) in marked contrast to the poor $(8 \%)$ re-association kinetics of total DNA (Brenner, 1978).

Early studies established the apparently universal occurrence of $23 \mathrm{~S}, 16 \mathrm{~S}$ and $5 \mathrm{~S}$ components of prokaryotic rRNA (Brenner et al., 1969). Thus, an observation by Winkler (1979) that at least one apparently wild-type strain of Salmonella typhimurium lacked intact $23 \mathrm{~S}$ rRNA was somewhat unexpected. We have therefore surveyed a collection of naturally occurring isolates of S. typhimurium, other serotypes of Salmonella and other Enterobacteriaceae, to determine how widespread this variation in rRNA might be and whether it was restricted to the strain studied by Winkler. The results of this kind of survey may, of course, have considerable potential for diagnostic purposes. Furthermore, we have investigated the cause of this difference in rRNA patterns and have shown that

Received 30 Nov. 1987; accepted 7 Dec. 1987. it results from differences in the rRNA genes themselves, rather than from differences in specific ribonucleases in the different species. The evolutionary implications of this finding are considered.

\section{Materials and methods}

\section{Bacteria}

The 65 strains of Enterobacteriaceae examined were: 15 clinical and laboratory-derived strains of Escherichia coli; 1 strain each of Shigella dysenteriae, Sh. boydii and Sh. flexneri and 2 strains of Sh. sonnei; two strains of Citrobacter freundii; 14 strains of $S$. typhimurium isolated from a diverse range of animal and environmental sources and 2 laboratory-maintained strains LT2 and LT7 (Lilleengen, 1948) that had been investigated by Winkler (1979); 7 strains of $S$. paratyphi $B ; 15$ other strains of Salmonella from subspecies I representing 12 different Oantigen groups and 15 host-specific or non-specific serotypes ( $S$. alachua, $S$. anatum, $S$. choleraesuis, $S$. enteritidis, S. hadar, S. heidelberg, S. infantis, S. kedougou, $S$. livingstone, $S$. paratyphi $A, S$. pomona, $S$. rubislaw, $S$. senftenberg, $S$. virchow and $S$. waycross); and 5 strains of Salmonella representing serotypes from subspecies II-V (S. II manombo, S. III arizonae [44: $\left.\mathrm{z}_{4}, \mathrm{z}_{24} ;-\right], S$. III arizonae $\left[48: \mathrm{i} ; \mathrm{z}_{35}\right], S . I V$ houten and $S . V$ bongor). The designation of serotypes of Salmonella in subspecies I-V follows recommended guidelines (Lindberg and Le Minor, 1984). Strains were stored on Dorset's egg slopes at room temperature until they were subcultured on MacConkey's Agar (Oxoid) for testing. 


\section{Media and bacterial growth}

L-broth (Miller, 1972) contained (/L), Bacto-Tryptone (Difco) $10 \mathrm{~g}$, Yeast Extract (Oxoid) $5 \mathrm{~g}$, and $\mathrm{NaCl} 5 \mathrm{~g}$. A single colony of each strain was inoculated to $5 \mathrm{ml}$ of Lbroth and incubated overnight at $37^{\circ} \mathrm{C}$ with vigorous shaking at $100 \mathrm{rpm}$. The overnight culture was diluted 1 in 50 in fresh L-broth $(10 \mathrm{ml})$ and the cells were grown to late exponential phase.

\section{Preparation of RNA for electrophoresis}

Cultures, grown as described above, were chilled and the cells pelleted by centrifugation at $6000 \mathrm{~g}$ for $10 \mathrm{~min}$. Where appropriate (vide infra), cultures of different strains were mixed in equal volumes before centrifugation. Bacterial cell pellets were washed in cold TrisEDTA buffer, $p \mathrm{H} 8.0$ (Maniatis et al., 1982) and lysed by the addition of $800 \mu \mathrm{l}$ of lysis buffer $(10 \mathrm{~mm}$ Tris- $\mathrm{HCl}, p \mathrm{H}$ 8.0 , containing $1 \mathrm{mM}$ EDTA, $350 \mathrm{mM} \mathrm{NaCl}$ and SDS $2 \% \mathrm{w} / \mathrm{v}$ ) and boiled for $1 \mathrm{~min}$. After mixing, $200 \mu \mathrm{l}$ of equilibrated phenol (Maniatis et al., 1982) was added and the lysate heated at $90^{\circ} \mathrm{C}$, with mixing, for a further $30 \mathrm{~s}$. Samples were chilled and extracted with $200 \mu \mathrm{l}$ of chloroform: isoamylalcohol (24:1). After centrifugation (12000 $\mathrm{g}$ for $4 \mathrm{~min}$ ), the supernate was removed and subjected to a further hot phenol-chloroform extraction and two extractions with chloroform-isoamylalcohol; the nucleic acids were precipitated twice with ethanol. Samples were resuspended in $50 \mu \mathrm{l}$ of TE buffer, denatured by addition of $20 \mu \mathrm{l}$ of gel-running buffer $(5 \times$ strength), $100 \mu \mathrm{l}$ of deionised formamide and $34 \mu \mathrm{l}$ of deionised formaldehyde $(36 \% \mathrm{w} / \mathrm{v})$, and heated at $55^{\circ} \mathrm{C}$ for $15 \mathrm{~min}$ with occasional mixing. One-tenth volume of loading dye (glycerol $50 \% \mathrm{v} / \mathrm{v}$ with $1 \mathrm{~mm}$ EDTA and bromophenol blue $0.4 \%$ w/v) was added and the RNA separated by electrophoresis.

\section{Formaldehyde-agarose gels}

Denaturing agarose gels $1.2 \% \mathrm{w} / \mathrm{v}$ (Maniatis et al., $1982)$ were prepared in gel-running buffer (0.04 M MOPS,

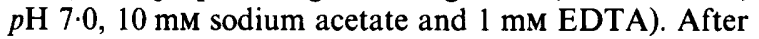
cooling the dissolved agarose to $55^{\circ} \mathrm{C}$, deionised formaldehyde was added to a final concentration of $2.2 \mathrm{M}$ and ethidium bromide at $0.5 \mu \mathrm{g} / \mathrm{ml}$. Gels were electrophoresed in the gel-running buffer described above at $5 \mathrm{~V} / \mathrm{cm}$ for $c .2 \mathrm{~h}$.

\section{Transformation of S. typhimurium strain LT2 with $r r n B$ genes of $E$. coli}

Plasmid pC6 encodes the entire $E$. coli $r r n B$ operon and is similar to pKK3535 (Brosius et al., 1981) but with the insert in the opposite orientation. This plasmid was kindly provided by $\mathrm{Dr} C$. Squires. The entire nucleotide sequence of the $r r n B$ operon is known (Brosius et al., 1981). To introduce this plasmid into S. typhimurium, pC6 DNA was first transformed into the restrictiondeficient strain LR5000 by the $\mathrm{CaCl}_{2}$ procedure (Leder- berg and Cohen, 1974) selecting for ampicillin resistance. DNA purified from this strain was subsequently transformed into the wild-type strain LT2 of $S$. typhimurium.

\section{Results}

\section{Absence of 23S rRNA from Salmonella}

When RNA preparations from cultures of 15 strains of E. coli, 5 strains of Shigella and 2 strains of Citrobacter were analysed by electrophoresis in denaturing formaldehyde-agarose gels, they displayed typical " $E$. coli" rRNA patterns with both $23 \mathrm{~S}$ and $16 \mathrm{~S}$ components (fig. 1). In contrast, all 16 strains of $S$. typhimurium examined, irrespective of their source or year of isolation, exhibited a very different pattern (the " $S$. typhimurium" pattern). Although the 16S rRNA band of $S$. typhimurium strains always migrated at the same rate as the $16 \mathrm{~S}$ band of $E$. coli, the strains of $S$. typhimurium lacked a band of mobility equivalent to the 23S rRNA of E. coli (fig. 1). In place of the $23 \mathrm{~S}$ rRNA band, two bands of mobility corresponding to 1.7 and $1 \cdot 1$ kilobases were observed. Within the genus Salmonella, all $S$. typhimurium strains gave the $S$. typhimurium rRNA pattern whilst all 20 strains from Salmonella subspecies I-V gave the $E$. coli pattern. Thus, the absence of a 23S rRNA seems to be specific to $S$. typhimurium. The only exceptions to this finding were 2 strains of $S$. paratyphi $B$ which showed the $S$.typhimurium pattern although 5 other strains of that serotype gave the normal $E$. coli pattern. The taxonomic significance of this observation is discussed below.

In $S$. typhimurium strains, the $23 \mathrm{~S}$ rRNA is replaced by two smaller rRNA species which were shown to be equivalent to 23S rRNA because they hybridised in "Northern" blots with plasmid pC6 DNA, encoding the 23S rRNA gene of $E$. coli (data not shown). To show that cleavage of 23S rRNA into two fragments reflected the situation in vivo, and was not due to cleavage during RNA extraction from $S$. typhimurium by nucleases active during the extraction process, equal volumes of cultures of $E$. coli and $S$. typhimurium were mixed immediately before cell lysis and RNA extraction. The mixed culture gave a mixed rRNA pattern (fig. 2) showing that Salmonella nucleases were not active during extraction.

The difference in rRNA patterns between $E$. coli and $S$. typhimurium might reflect a difference in intracellular RNAases, such that $S$. typhimurium contains an RNAase that cleaves 23S rRNA but is absent from $E$. coli. Alternatively, the nucleases of 

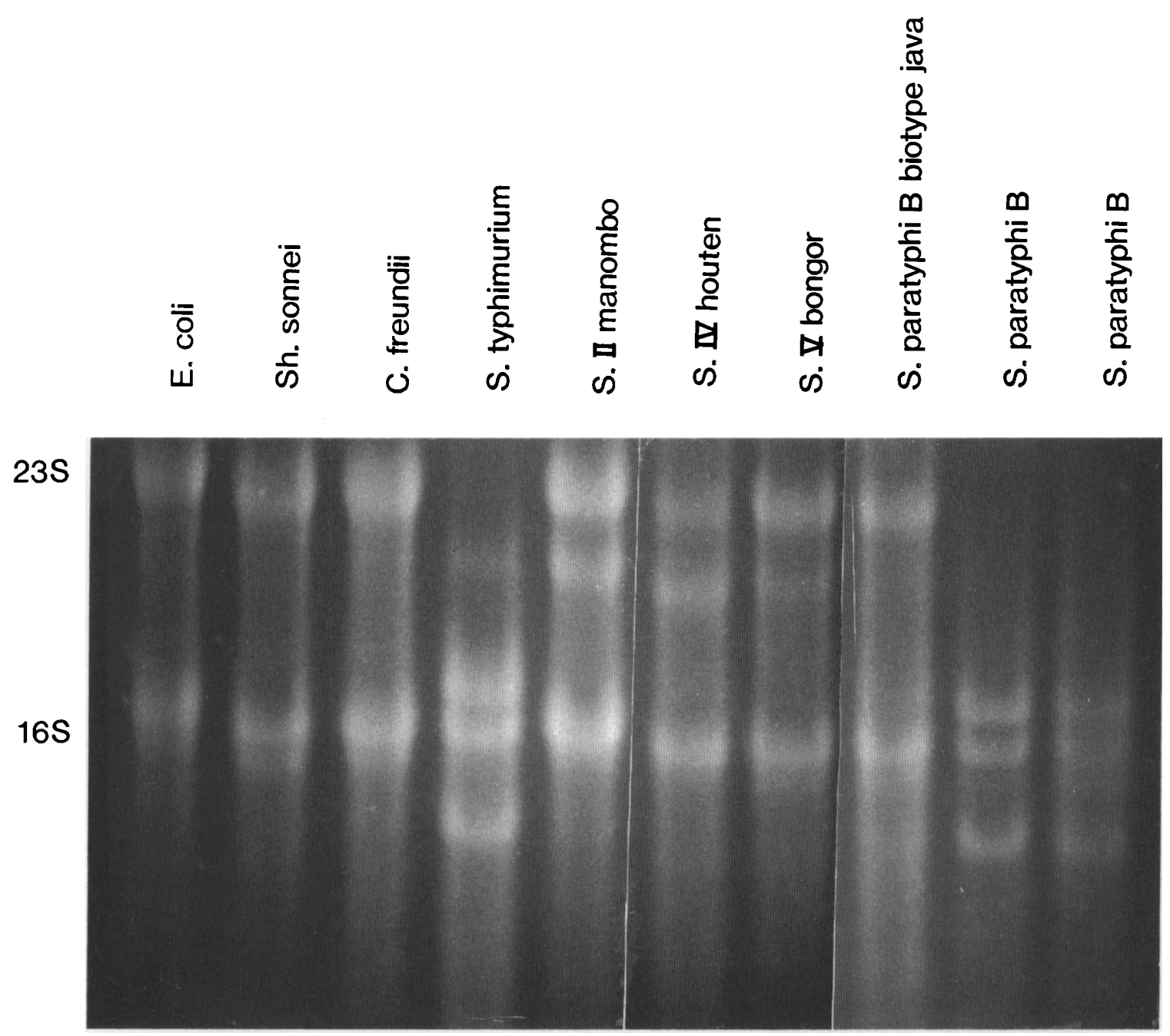

Fig. 1. Agarose gel showing rRNA patterns from various Enterobacteriaceae. The 23S and 16S rRNA species are indicated. Details of the strains are given in the text.

the two species may be the same, but a sequence difference in the rRNA makes $S$. typhimurium $23 S$ rRNA far more susceptible to cleavage. To address this question plasmid pC6, encoding the $E$. coli $23 \mathrm{~S}$ rRNA, was transformed into $S$. typhimurium strain LT2 and the rRNA extracted and separated by electrophoresis (fig. 3). The E. coli 23S rRNA remained intact when expressed in $S$. typhimurium demonstrating that the cleavage of $S$. typhimurium 23S rRNA resulted from sequence differences in the rRNA molecules rather than a difference in the specificity of the nucleases in the two species.

\section{Discussion}

The introduction of numerical taxonomy and the technique of DNA-DNA re-association has re- sulted in the description of many new species and genera in the family Enterobacteriaceae, which now includes more than 125 hybridisation groups (Brenner, 1983; Farmer et al., 1985; Ewing, 1986). It has also indicated the need for a reappraisal of some of the accepted relationships implied from earlier taxonomic schemes which were devised on the basis of only a few phenotypic traits. It might be useful, therefore, to consider the genetic relatedness of the Enterobacteriaceae used in this study.

First, although the four species of Shigella were originally delineated from $E$. coli on the basis of their pathogenicity, and have been maintained as separate entities in the interests of clinical microbiology and epidemiology (Ewing, 1986), they nevertheless constitute with $E$. coli a single genetic species (i.e., they show $\geqslant 75 \%$ DNA relatedness 


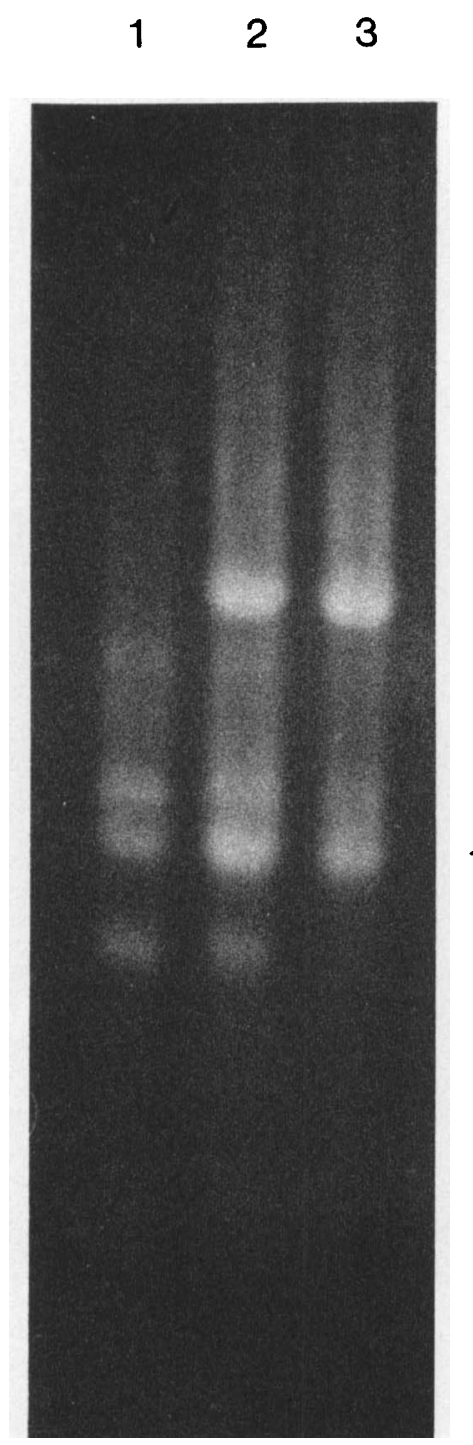

Fig. 2. Agarose gel showing rRNA from: track 1, S. typhimurium strain LT2; track 2, S. typhimurium strain LT2 extracted together with $E$. coli strain K12; track $3, E$. coli strain K12. The E. coli 23S and 16S rRNA species are indicated.

under stringent conditions; Brenner et al., 1973). Second, the taxonomy of Salmonella has been similarly problematic. In this genus, strains differentiated by serotyping of $\mathrm{O}$ and $\mathrm{H}$ antigens were originally given species ranking; the eventual result of that approach was that $>2000$ serotypes masqueraded as species. Modern methods of taxonomy have now established that all strains of salmonellae and arizonae (the latter previously differentiated from the former by a few biochemical characters) constitute a single genetic species comprising seven

\section{$23 S$}

$16 S$

Fig. 3. Agarose gel showing rRNA from: track 1, S. typhimurium strain LT2; track 2, S. typhimurium strain LT2 transformed with plasmid pC6 encoding the $E$. coli $23 \mathrm{~S}$ rRNA. The $23 \mathrm{~S}$ and $16 \mathrm{~S}$ rRNA species are indicated.

subspecies (Le Minor et al., 1982 and 1986). The citrobacters, close relatives of the salmonellae, used to be included with them in the tribe Salmonelleae but are now known to have diverged sufficiently from them to warrant distinct taxonomic status in the tribe Citrobactereae (Ewing, 1986). Furthermore, citrobacters afford a useful link between escherichiae and salmonellae because, in terms of DNA relatedness, species of Citrobacter are as closely related $(40-50 \%)$ to $E$. coli as they are to Salmonella (Crosa et al., 1973; Brenner, 1978).

Our findings show that strains of E. coli, Shigella, C. freundii and most serotypes of Salmonella give a similar rRNA pattern in which both $16 \mathrm{~S}$ and $23 \mathrm{~S}$ species of rRNA are represented. This commonality amongst four genera of Enterobacteriaceae which, in terms of DNA relatedness, might be considered to be no more than moderately related, only serves 
to highlight the unusual rRNA pattern of strains of $S$. typhimurium. The 14 naturally occurring strains of $S$. typhimurium examined by us were isolated between 1950 and 1985 from diverse human, animal and environmental sources in 12 different countries worldwide. They represent 14 of the phage-type/ biotype groups described by Anderson et al. (1978). Genetic data reported elsewhere (Old, 1984) suggest that strains of $S$. typhimurium of all phage-type/ biotype groups evolved from a common, archetypal bacterium. The present findings, reporting an absence of 23S rRNA from all strains of $S$. typhimurium examined by us, support this hypothesis. In contrast, our observation that some strains of $S$. paratyphi $B$ possess the $E$. coli rRNA pattern, whereas others possess the $S$. typhimurium pattern, does not fit the classical taxonomic grouping. This finding implies that certain members of the $S$. paratyphi $B$ serotype would best be classified as $S$. typhimurium. The need to reassess the classification of $S$. paratyphi $B$ is not inconsistent with the known heterogeneity of strain characters in that serotype and is discussed more fully by Barker et al. (1988).

The finding that a wide range of Salmonella strains lacks a $23 \mathrm{~S}$ rRNA species is somewhat surprising in view of the conserved nature of rRNA sequences among species and the essential role of

\section{REFERENCES}

Anderson E S, Ward L R, de Saxe M J, Old D C, Barker R, Duguid J P 1978 Correlation of phage type, biotype and source in strains of Salmonella typhimurium. Journal of Hygiene 81 : 203-217.

Barker R M, Kearney G M, Nicholson P, Blair A L, Porter R C, Crichton P B 1988 Types of Salmonella paratyphi B and their phylogenetic significance. Journal of Medical Microbiology 26: 285-294.

Brenner D J 1978 Characterization and clinical identification of Enterobacteriaceae by DNA hybridization. Progress in Clinical Pathology 7 : 71-117.

Brenner D J 1983 Impact of modern taxonomy on clinical microbiology. ASM News 49: 58-63.

Brenner D J, Falkow S 1971 Genetics of the Enterobacteriaceae C. Molecular relationships among members of the Enterobacteriaceae. Advances in Genetics 16: 81-118.

Brenner D J, Fanning G R, Miklos G V, Steigerwalt A G 1973 Polynucleotide sequence relatedness among Shigella species. International Journal of Systematic Bacteriology 23: 17.

Brenner D J, Fanning G R, Rake A V, Johnson K E 1969 A batch procedure for thermal elution of DNA from hydroxyapatite. Analytical Biochemistry 28: 447-459.

Brosius J, Dull T J, Sleeter D D, Noller H F 1981 Gene organization and primary structure of a ribosomal RNA operon from Escherichia coli. Journal of Molecular Biology 148: $107-127$. this RNA in ribosome function. We have shown that the lack of 23S rRNA in S. typhimurium is not an artefact of rRNA isolation. Furthermore, when the $E$. coli rrnB gene is expressed in $S$. typhimurium the 23S rRNA remains intact, implying that the difference between $E$. coli and $S$. typhimurium is not a specific ribonuclease activity. Rather, there must be a sequence difference in the $23 \mathrm{~S}$ rRNA from the two species. This is an intriguing possibility in view of the fact that the rRNAs of $E$. coli and $S$. typhimurium are transcribed from seven independent $r r n$ operons (Lehner et al., 1984). Since all the 23S rRNA molecules of S. typhimurium are cleaved, this implies that each of the seven $r r n$ genes has acquired the same alteration. Presumably, gene conversion events have ensured that, within a species, the seven genes are maintained in the same genetic configuration. This provides what is probably the best evidence for gene conversion in prokaryotes and defines an interesting evolutionary system for studying the mechanisms of gene conversion.

We are grateful to Dr Cathy Squires for providing plasmid pC6 and to Dr Ruth Barker for useful discussions. The work was funded by an MRC grant to $\mathrm{CFH}$ who is a Lister Institute Research Fellow, and by a grant from the University of Dundee Research Initiatives Fund to DCO.
Crosa J N, Brenner D J, Ewing W H, Falkow S 1973 Molecular relationships among the Salmonelleae. Journal of Bacteriology $115: 307-315$.

Ewing W H 1986 Edwards and Ewing's Identification of Enterobacteriaceae, 4th edn. Elsevier Science Publishing Co. Inc., New York.

Farmer J J et al. 1985 Biochemical identification of new species and biogroups of Enterobacteriaceae isolated from clinical specimens. Journal of Clinical Microbiology 21 : 46-76.

Kohne D E 1968 Isolation and characterization of bacterial ribosomal RNA cistrons. Biophysical Journal 8: 1104-1118.

Lederberg E M, Cohen S N 1974 Transformation of Salmonella typhimurium by plasmid deoxyribonucleic acid. Journal of Bacteriology 119: 1072-1074.

Lehner A F, Harvey S, Hill C W 1984 Mapping and spacer identification of rRNA operons of Salmonella typhimurium. Journal of Bacteriology 160: 682-686.

Le Minor L, Véron M, Popoff M 1982 Taxonomie des Salmonella. Annales de Microbiologie (Paris) 133B: 223-243.

Le Minor L, Popoff M Y, Laurent B, Hermant D 1986 Individualisation d'une septième sous-espèce de Salmonella: $S$. choleraesuis subsp. indica subsp. nov. Annales de Microbiologie (Paris) 137B: 211-217.

Lilleengen K 1948 Typing of Salmonella typhimurium by means of bacteriophage. Acta Pathologica et Microbiologica Scandinavica 77 Suppl. : 1-125.

Lindberg A A, Le Minor L 1984 Serology of Salmonella. In: Bergan T (ed) Methods in microbiology, vol. 15. Academic Press, London, pp 1-141. 
Maniatis T, Fritsch, E F, Sambrook J 1982 Molecular cloning. A laboratory manual. Cold Spring Harbor Laboratory, Cold Spring Harbor, New York.

Miller J H 1972 Experiments in molecular genetics. Cold Spring Harbor Laboratory, Cold Spring Harbor, New York.
Old D C 1984 Phylogeny of strains of Salmonella typhimurium. Microbiological Sciences 1 : 69-72.

Winkler M E 1979 Ribosomal ribonucleic acid isolated from Salmonella typhimurium: absence of the intact $23 \mathrm{~S}$ species. Journal of Bacteriology 139: 842-849. 